\title{
Elastic and Acoustic Properties of Heavy Rare-Earth Metals
}

\author{
Pramod Kumar Yadawa ${ }^{*}$, , Devraj Singh ${ }^{1}$, Dharmendra Kumar Pandey ${ }^{2}$ and Raja Ram Yadav ${ }^{3}$ \\ ${ }^{1}$ Department of Applied Physics, AMITY School of Engineering and Technology, Bijwasan, New Delhi-110 061, India \\ ${ }^{2}$ Department of Physics, P.P.N. College, Kanpur-208 001, India \\ ${ }^{3}$ Department of Physics, University of Allahabad, Allahabad-211 002, India
}

\begin{abstract}
In this paper, ultrasonic properties like ultrasonic attenuation, sound velocities, acoustic coupling constants and thermal relaxation time have been studied in hexagonal structured metals Gadolinium (Gd), Terbium (Tb), Dysprosium (Dy), Holmium (Ho), Erbium (Er) and Thulium (Tm) along unique axis at room temperature. For the evaluations of ultrasonic properties, second- and third- order elastic constants have been computed also. The peculiar behavior of these metals is found at $55^{\circ}$ due their least thermal relaxation time and highest Debye average velocity. Dy is more ductile, stable, perfect metal in comparison to other chosen metals due to its lowest value of attenuation. So we predict that Dy is most suitable lanthanide metals for material science and engineering.
\end{abstract}

\section{INTRODUCTION}

Ultrasonic nondestructive testing is a resourceful technique that can be applied for investigation of several materials. This is useful for characterization of microstructures, appraisal of defects, and evaluation of objects properties. By virtue of this, ultrasonic measurements during fabrication and heat treatment allow ensuring the absence of unacceptable discontinuities and the presence of a particular microstructure with preferred properties. The interaction of ultrasound with microstructure is important for many material problems. Attenuation and backscattering reduce the delectability of flaws, specially in materials with coarse grains or complex microstructures such as platinum alloys. Further, quantification of these wave propagation properties provide information about the microstructure that can be used in materials description studies, e.g. nondestructive determination of grain size. Acoustic coupling constant (Non-linearity parameter) plays vital role for conversion of acoustical energy into thermal energy via phonon-phonon interaction in materials science. This property of material is very interested for material scientists since last decade. Wave propagation velocity is another key factor in ultrasonic characterization, which in combination with attenuation can provide important tools in understanding, the inspectability of materials; for example, it can provide information about crystallographic texture. Ultrasonic velocity is directly related to elastic constants i.e. $V=\sqrt{ }(C / \rho)$, where $C$ is the elastic constant and $\rho$, the density of that particular material. The elastic constants, in particular, provide valuable information on the stability and stiffness of materials [1-4]. Grüneisen parameter is direct

*Address correspondence to this author at the Department of Applied Physics, AMITY School of Engineering and Technology, Bijwasan, New Delhi-110 061, India; E-mail: pkyadawa@aset.amity.edu consequence of second and third order elastic constants as given in Appendix.

The important source of the lanthanides is monazite. Like most metals, the lanthanides have a bright silvery appearance. The lanthanides form alloys (mixtures) with many other metals, and these alloys exhibit a wide range of physical properties. The lanthanides react slowly with cold water and more rapidly with hot water to form hydrogen gas, while burn readily in air to form oxides. They also form compounds with many nonmetals, such as hydrogen, fluorine, phosphorous, sulfur, and chlorine. Gadolinium is a soft silvery-white metal that is used as an alloying agent in some steels and in the manufacture of some electronic components [5]. The lanthanides are used as catalyst to speed up chemical reactions, refining agent for the conversion of crude oil into gasoline, kerosene and diesel. These are also used as phosphors in color television sets. Other lanthanide compounds are used in streetlights, searchlights, and in the high-intensity lighting present in sports stadiums. The behavior of the elastic moduli in the vicinity of a second-order phase transition was described elsewhere. Landau et al. showed that the ultrasonic attenuation should display a maximum at the low temperature side of the transition point [6].

In the present investigation, the ultrasonic properties like attenuation, sound velocities, acoustic coupling constants \& thermal relaxation time and higher order elastic constants of $\mathrm{Gd}, \mathrm{Tb}, \mathrm{Dy}, \mathrm{Ho}, \mathrm{Er}$ and Tm metals at room temperature have been investigated for their characterization at $300 \mathrm{~K}$ using modified Mason's approach. With the knowledge of the authors, no study has been made for h.c.p. structured metals using simultaneously both elastic constants and attenuation theories. The obtained results are discussed in correlation with known physical properties. 


\section{THEORY}

\subsection{Higher Order Elastic Constants}

The second $\left(\mathrm{C}_{\mathrm{IJ}}\right)$ and third $\left(\mathrm{C}_{\mathrm{IJK}}\right)$ order elastic constants of material are defined by following expressions.

$C_{I J}=\frac{\partial^{2} U}{\partial e_{I} \partial e_{J}} ; \quad$ I or $\mathrm{J}=1, \ldots \ldots 6$

$C_{I J K}=\frac{\partial^{3} U}{\partial e_{I} \partial e_{J} \partial e_{K}} ;$ I or $\mathrm{J}$ or $\mathrm{K}=1, \ldots \ldots 6$

where, $U$ is elastic energy density, $e_{I}=e_{i j}$ ( $i$ or $j=x, y, z, I=1$, ...6) is component of strain tensor. Eqs. (1)-(2) leads six second and ten third order elastic constants (SOEC and TOEC) for the hexagonal closed packed structured materials $[7]$.

$$
\left.\begin{array}{lrl}
C_{11}=24.1 \mathrm{p}^{4} C^{\prime} & C_{12}=5.918 \mathrm{p}^{4} C^{\prime} \\
C_{13}=1.925 \mathrm{p}^{6} C^{\prime} & C_{33}=3.464 \mathrm{p}^{8} C^{\prime} \\
C_{44}=2.309 \mathrm{p}^{4} C^{\prime} & C_{66}=9.851 \mathrm{p}^{4} C^{\prime}
\end{array}\right\}
$$

where $\quad C^{\prime}=\chi \mathrm{a} / \mathrm{p}^{5}$;

$$
B=\psi \mathrm{a}^{3} / p^{3}
$$

$\chi=(1 / 8)\left[\left\{n b_{0}(n-m)\right\} /\left\{\mathrm{a}^{\mathrm{n}+4}\right\}\right]$

$\psi=-\chi /\left\{6 \mathrm{a}^{2}(m+n+6)\right\} ; \quad m, \quad n=$ integer quantity; $b_{0}=$ Lennard Jones parameter. $p=c / a$ : axial ratio; $c$ is the height of the unit cell and $a$ be the basal plane distance.

\subsection{Ultrasonic Velocity}

The anisotropic behavior of the material can be understood with the knowledge of ultrasonic velocity because the velocity is related to the second order elastic constants [7]. On the basis of mode of atomic vibration, there are three types of velocities (longitudinal, quasi shear and shear) in acoustical region [8]. These velocities vary with the direction of propagation of wave from the unique axis of hexagonal structured crystal [9]. The ultrasonic velocities as a function of angle between direction of propagation and unique axis for hexagonal structured materials are [10]:

$$
\begin{aligned}
V_{L}^{2}=\{ & C_{33} \operatorname{Cos}^{2} \theta+C_{11} \operatorname{Sin}^{2} \theta+C_{44}+ \\
& \left\{\left[C_{11} \operatorname{Sin}^{2} \theta-C_{33} \operatorname{Cos}^{2} \theta+C_{44}\left(\operatorname{Cos}^{2} \theta-\operatorname{Sin}^{2} \theta\right)\right]^{2}\right. \\
& \left.\left.+4 \operatorname{Cos}^{2} \theta \operatorname{Sin}^{2} \theta\left(\mathrm{C}_{13}+C_{44}\right)^{2}\right\}^{1 / 2}\right\} / 2 \rho \\
V_{S 1}^{2}= & \left\{C_{33} \operatorname{Cos}^{2} \theta+C_{11} \operatorname{Sin}^{2} \theta+C_{44}\right. \\
& -\left\{\left[C_{11} \operatorname{Sin}^{2} \theta-C_{33} \operatorname{Cos}^{2} \theta+C_{44}\left(\operatorname{Cos}^{2} \theta-\operatorname{Sin}^{2} \theta\right)\right]^{2}\right. \\
& \left.\left.+4 \operatorname{Cos}^{2} \theta \operatorname{Sin}^{2} \theta\left(\mathrm{C}_{13}+C_{44}\right)^{2}\right\}^{1 / 2}\right\} / 2 \rho \\
V_{S 2}^{2}= & \left\{C_{44} \operatorname{Cos}^{2} \theta+C_{66} \operatorname{Sin}^{2} \theta\right\} / \rho
\end{aligned}
$$

where $V_{L}, V_{S 1}$ and $V_{S 2}$ are longitudinal, quasi shear and pure shear wave ultrasonic velocities. Variables $\rho$ and $\theta$ represent the density of the material and angle with the unique axis of the crystal respectively. The Debye temperature $\left(T_{D}\right)$ is an important physical parameter for the characterization of materials, which is well related to the Debye average velocity $\left(V_{D}\right)$.

$T_{D}=\frac{\hbar \mathrm{V}_{\mathrm{D}}\left(6 \pi^{2} \mathrm{n}_{\mathrm{a}}\right)^{1 / 3}}{k_{B}}$

here $\quad V_{D}=\left\{\frac{1}{3}\left(\frac{1}{V_{L}^{3}}+\frac{1}{V_{S 1}^{3}}+\frac{1}{V_{S 2}^{3}}\right)\right\}^{-1 / 3}$

where $\hbar$ is quantum of action and is equal to Planck's constant divided by $2 \pi ; k_{B}$ is Boltzmann Constant; $n_{a}$ is atom concentration.

When the ultrasonic wave propagates through crystalline material, the equilibrium of phonon distribution is disturbed. The time for re-establishment of equilibrium of the thermal phonon distribution is called thermal relaxation time $(\tau)$. In the process of re-establishment, thermal energy is interchanged among various modes. Hence the rate, at which interchange of thermal energy to diverse modes, is called as thermal relaxation time. and is correlated with the Debye average velocity.

$\tau=3 \mathrm{~K} / C_{V} V_{D}^{2}$

here $K$ and $C_{V}$ are the thermal conductivity and specific heat per unit volume of the material respectively.

The net attenuation rate is governed by the rate at which energy is removed by the transverse waves to the highfrequency thermal waves. Since half of the built-up low frequency phonons are shear and half longitudinal. Only shear phonons can convert their energy to high-frequency thermal waves, thus the relaxation time for longitudinal waves $\left(\tau_{L}\right)$ is twice the thermal relaxation time $\left(\tau_{t h}\right)$, and relaxation time for shear waves $\left(\tau_{S}\right)$ is equal to thermal relaxation time i.e. $\tau_{S}=\tau_{L} / 2[4]$.

The above formulae have been used for the evaluation of ultrasonic velocity and related parameters for the selected materials.

\subsection{Acoustic Coupling Constant}

The Akhieser effect is an energy dissipation effect taking place under a uniform time dependent stress. The propagation of an ultrasonic wave produces a stress in crystalline material, which results strain. An incremental change in temperature is caused by alternating strain. This increment in temperature disturbs the equilibrium of phonon distribution, which is equalized to the equilibrium through relaxation by means of phonon-phonon collision causing attenuation of wave. Under consideration of anisotropic behavior of crystalline material (non-linear higher order elastic constant), there is a change in elastic moduli ( $\Delta C$ ) caused by separation of phonon modes from equilibrium due 
to increment in temperature affected with the propagation of wave (time dependent stress) [4].

$$
\Delta C=\left(3 E_{0}<\left(\gamma_{I}^{J}\right)^{2}>-<\gamma_{I}^{J}>^{2} C_{V} T\right)
$$

here $E_{0}, C_{V}$ and $\mathrm{T}$ are the thermal energy density, specific heat per unit volume and required temperature respectively. $\gamma_{I}^{J}$ is known as Grüneisen number; where I and $\mathrm{J}$ denote the mode of vibration and direction of propagation respectively. The ratio of $3 \Delta C$ and $E_{0}$ is called as non-linearity parameter or acoustic coupling constants $(D)$, which directly measures the amount of ultrasonic energy converted into thermal energy by the process of relaxation. The acoustic coupling constants would be expressed as:

$$
D=3\left(3 E_{0}<\left(\gamma_{I}^{J}\right)^{2}>-<\gamma_{I}^{J}>^{2} C_{V} T\right) / E_{0}
$$

This physical parameter is a dominating factor for ultrasonic attenuation in hexagonal structured materials [11]. The Grüneisen number $\left(\gamma_{I}^{J}\right)$ is direct consequence of higher order elastic constants, which depends mode of vibration and direction of propagation. The expressions of Grüneisen number for the wave propagation along the unique axis of hexagonal structured materials are listed in Appendix. Akhieser damping is directly related to acoustic coupling constant, higher order elastic constants, density and thermal relaxation time. The ultrasonic attenuation caused by the Akhieser damping $\left\{\left(\mathcal{A} / \mathrm{f}^{2}\right)_{\text {Akh }}\right\}$ can be calculated using formulae given in our previous paper [9].

\section{RESULTS}

The unit cell parameters ' $a$ ' and ' $p$ ' for Gd, Tb, Dy, Ho, Er and $\mathrm{Tm}$ are $3.63 \AA, 3.60 \AA, 3.59 \AA, 3.58 \AA, 3.56 \AA, 3.54 \AA$ and $1.592,1.583,1.574,1.570,1.570,1.571$ respectively. The value of $m, n$ and $b_{0}$ for these lanthanides metals are 6, 7 and $2.3 \times 10^{-64} \mathrm{erg} \mathrm{cm}^{7}$ correspondingly. The second and third order elastic constants have been calculated for lanthanide metals using Eqs. (3a and 3b) and are presented in Table 1.

The density $(\rho)$ and thermal conductivity $(K)$ at room temperature has been taken from the literature $[11,13]$. The value of specific heat per unit volume $\left(C_{V}\right)$ and thermal energy density $\left(E_{0}\right)$ have been evaluated using tables of physical constants and calculated Debye temperature. The angle dependent $V_{D}$ and thermal relaxation time have been evaluated with Eqs. (8) and (9), which are shown in Figs. (1, 2). The quantities $K$ and calculated acoustic coupling constants $\left(D_{L} \& D_{S}\right)$ are presented in Table 2 . The calculated ultrasonic attenuation coefficient over frequency square for longitudinal $\left\{\left(A / f^{2}\right)_{L}\right\}$ and shear $\left\{\left(A / f^{2}\right)_{S}\right\}$ waves due p-p interaction under condition $\omega \tau<<1$ is presented in Table 2 . The evaluated Debye temperature and Debye average velocity for propagation of wave along unique axis of chosen crystals are visualized in Fig. (3).

\section{DISCUSSION}

The elastic constants are important since they are related to hardness and are used for the determination of the

Table 1. Second and Third Order Elastic Constants $\left(\right.$ in $\left.10^{10} \mathrm{Nm}^{-2}\right)$ of Metals at $300 \mathrm{~K}$

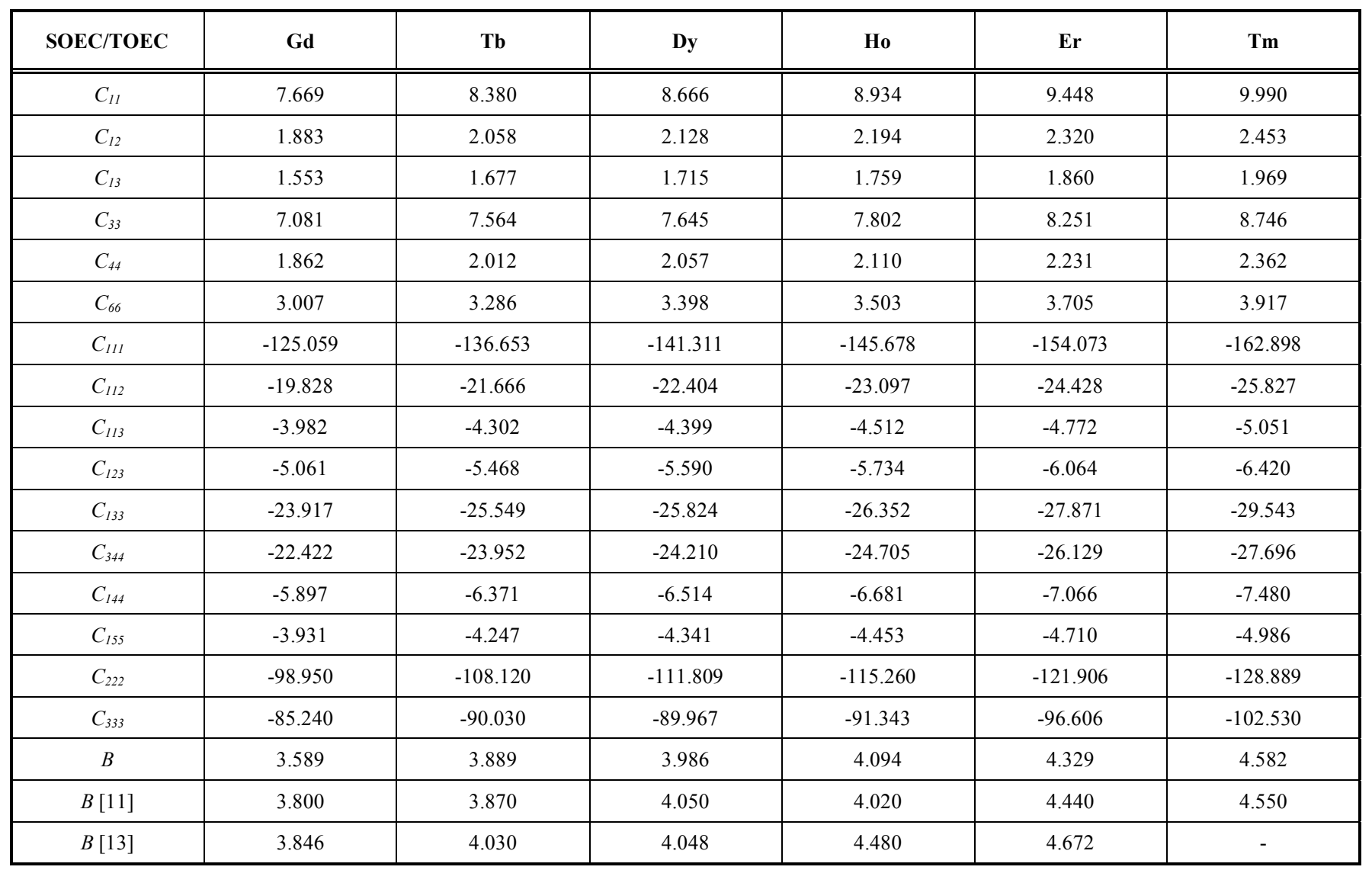


Table 2. Ultrasonic Velocities (in $\left.10^{3} \mathrm{~m} / \mathrm{s}\right)$, Thermal Conductivity $\left(K\right.$ : in $\left.\mathrm{Wm}^{-1} \mathrm{~K}^{-1}\right)$, Acoustic Coupling Constant $\left(D_{L}\right.$, $\left.D_{S}\right)$ and Ultrasonic Attenuation Coefficient (in $10^{-15} \mathrm{Nps}^{2} \mathrm{~m}^{-1}$ ) of Lanthanide Metals

\begin{tabular}{|c|c|c|c|c|c|c|}
\hline Property & Gd & Tb & Dy & Ho & Er & Tm \\
\hline \hline$K$ & 10.6 & 11.1 & 10.7 & 16.2 & 14.3 & 16.8 \\
\hline$D_{L}$ & 56.746 & 56.734 & 56.562 & 56.429 & 56.425 & 56.454 \\
\hline$D_{S}$ & 1.275 & 1.418 & 1.594 & 1.682 & 1.682 & 1.660 \\
\hline$\left(A / f^{2}\right)_{L}$ & 18.338 & 16.998 & 16.668 & 24.870 & 19.926 & 21.020 \\
\hline$\left(A / f^{2}\right)_{S}$ & 1.528 & 1.548 & 1.683 & 2.636 & 2.112 & 2.201 \\
\hline$\left(A / f^{2}\right)_{A k h}$ & 19.866 & 18.546 & 18.351 & 27.506 & 22.038 & 23.221 \\
\hline
\end{tabular}

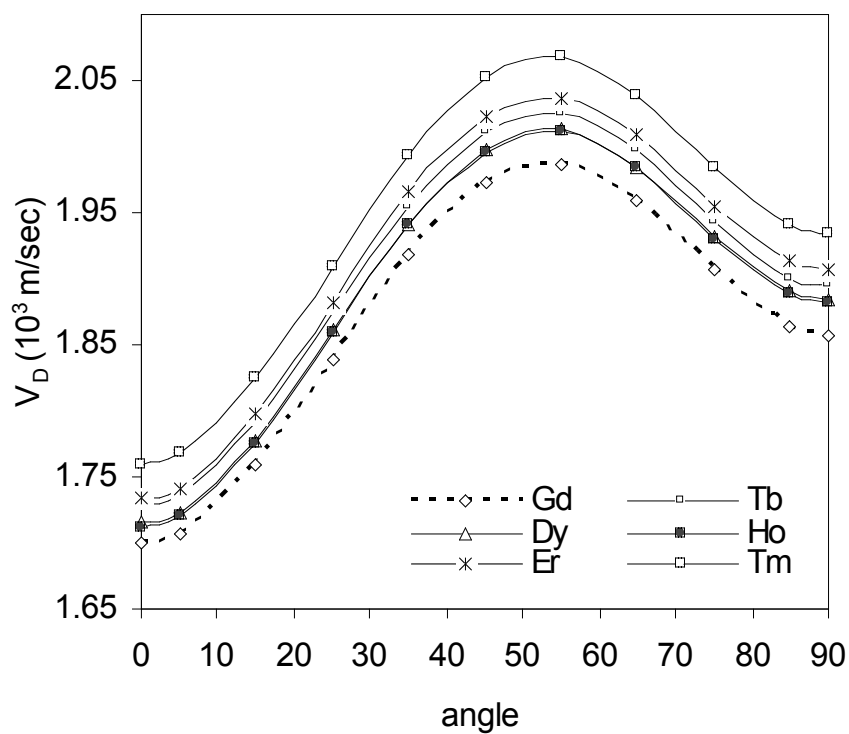

Fig. (1). $V_{D} v s$ angle with unique axis of crystal.

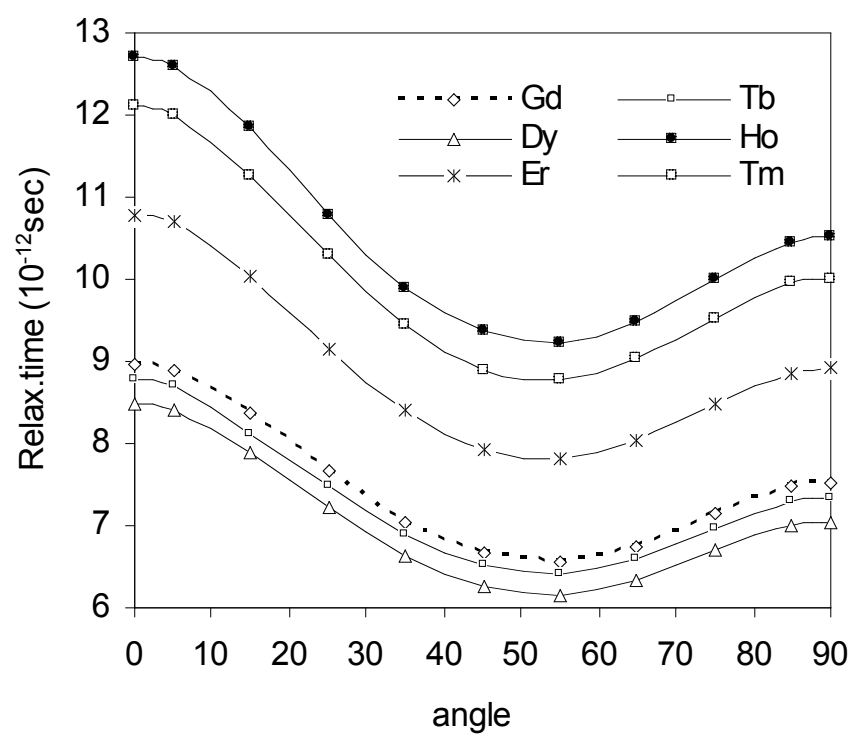

Fig. (2). Relax. time $v s$ angle with unique axis of crystal.

ultrasonic velocity. The bulk moduli of chosen metals are evaluated using the second order elastic constants. It is obvious from Table $\mathbf{1}$ that, there is good agreement between

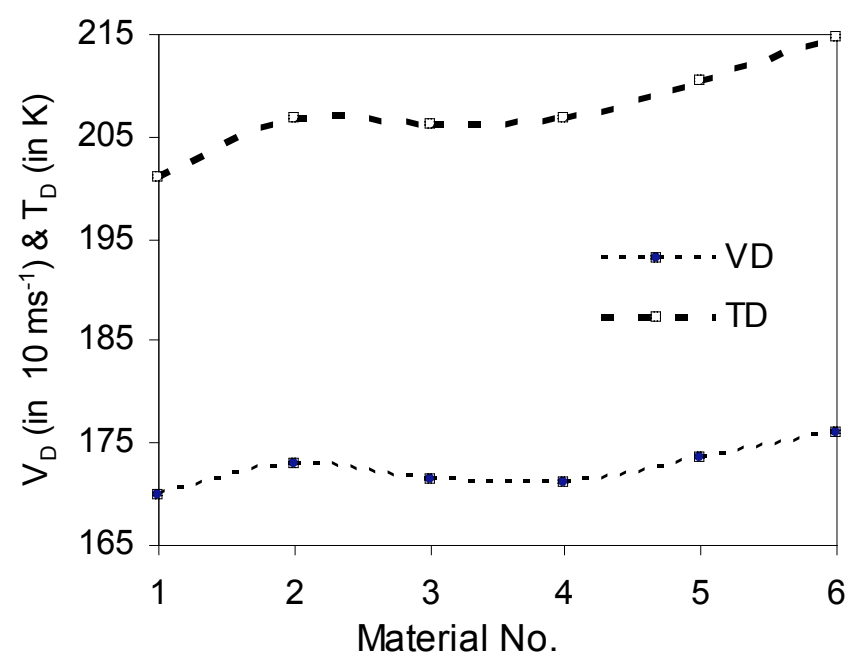

Fig. (3). $V_{D}$ and $T_{D}$ of materials (1.Gd, 2.Tb, 3.Dy, 4.Ho, 5.Er and 6.Tm).

the present and reported theoretical/experimental bulk moduli $[12,13]$. The experimental values of $C_{11}, C_{12}, C_{13}, C_{33}$ and $C_{44}$ for Dysprosium are $7.31 \times 10^{10} \mathrm{Nm}^{-2}, 2.53 \times 10^{10} \mathrm{Nm}^{-2}, 2.23$ $\mathrm{x} 10^{10} \mathrm{Nm}^{-2}, 7.81 \times 10^{10} \mathrm{Nm}^{-2}$ and $2.40 \times 10^{10} \mathrm{Nm}^{-2}$ respectively while the same for Holmium are $7.61 \times 10^{10} \mathrm{Nm}^{-2}, 2.48$ $\mathrm{x} 10^{10} \mathrm{Nm}^{-2}, 2.06 \times 10^{10} \mathrm{Nm}^{-2}, 7.76 \times 10^{10} \mathrm{Nm}^{-2}$ and $2.57 \times 10^{10} \mathrm{Nm}^{-2}$ [14]. Although these second order elastic constants have deviation of $10-20 \%$, but the order of the obtained SOEC is similar to experimental. In the experimental literature [14], the reported values of SOEC are at angle $45^{\circ}$ using acoustic wave velocities, in present investigation obtained results are of combined effect of all the angles. Hence applied theory for the evaluation of higher order elastic constants at room temperature is applicable for these metals. The SOEC are found to be increasing from metal $\mathrm{Gd}$ to $\mathrm{Tm}$. Thus the thermal softening behaviors are obtained as we move from Tm to Gd. These metals have low bulk modulus and higher order elastic constants in comparison to other hexagonal structured material [7-10,12, 15-17]. This reveals that large strain will develop for a given deforming force in these materials. A study of giant magnetostrictive effect of dysprosium and holmium indicates that for compensation of magnetic energy, large strain develops in these materials. Thus present analysis gives the satisfactory results with previous literature $[18,19]$. 
The ultrasonic velocities are evaluated using elastic constants and density which in turn provides Debye average velocity in all chosen materials. The plot of $V_{D}$ (Fig. 1) for the propagation of wave at different angles with unique axis implies that these metals have slight variation in velocity for each direction of propagation and have maxima at $\theta=55^{\circ}$. The angle variation of $V_{D}$ is similar with other hexagonal structured materials $[7,9,10,12]$. The combined effect of second order elastic constant and density causes the anomalous nature of angle dependent $V_{D}$ for the preferred metals.

It is reported that the magnetic behavior of Dy and Ho is simpler than other rare-earth heavy metals and have approximately same magnetic structure [14]. A similar conclusion can be drawn for Dy and Ho in present investigation. The $V_{D}$ is found approximately same for Dy and Ho; hence they have similar mechanical properties and can not be classified on the basis of Debye average velocity. Metals Gd and Tm have the lowest and the highest average sound velocity respectively. The higher order elastic constants and ultrasonic velocity of the chosen metals are comparable to the lave-phase compounds [12].

It is depicted from Fig. (2), the orders of relaxation time $(\tau)$ for these metals are also found in the order of pico second, which is the same as the other metals [20,21]. The value of relaxation time is found highest and lowest for Ho and Dy respectively in accordance with their thermal conductivity. Thus the minimum time is required for re-establishment of equilibrium distribution of thermal phonon in dysprosium. The relaxation time for all the metals is achieved maximum and minimum at angles $0^{\circ}$ and $55^{\circ}$ respectively, which is just converse behavior of Debye average velocity. This converse behavior of relaxation time validates the Eq. (9).

The Debye temperature of a material is a suitable parameter to describe the phenomenon of solid-state physics that are associated with lattice vibrations. In the conventional Debye model, $\mathrm{T}_{\mathrm{D}}$ can be defined by Eq. (7). From Eq. (7), the $T_{D}$ is directly proportional to $V_{D}$ [22]. It is obvious from Fig. (3) that the nature of Debye temperature and Debye average velocity is located similar. This is confirmation of Debye temperature computation.

The acoustic coupling constant (Table 2) for longitudinal wave is greater than that for shear wave as expected in literature $[20,21]$. The acoustic coupling constants for the longitudinal wave of these metals are found approximately five times larger than third group nitride [9]. So the conversion of acoustic energy into thermal energy in the present materials may be predicted to be large. In the evaluation of ultrasonic attenuation, it is supposed that wave is propagating along the unique axis $(<001>$ direction $)$ of lanthanide metals. The ultrasonic attenuation for longitudinal wave $\left(A / \mathrm{f}^{2}\right)_{\mathrm{L}}$ is greater than that for shear wave $\left(A / \mathrm{f}^{2}\right)_{S}$. This reveals that attenuation due to $\mathrm{p}-\mathrm{p}$ interaction for longitudinal wave is governing factor for Akhieser damping $\left\{\left(A / f^{2}\right)_{A k h}=\left(A / f^{2}\right)_{L}+\left(A / f^{2}\right)_{S}\right\}$. The ultrasonic attenuation due to $\mathrm{p}-\mathrm{p}$ interaction is mainly affected by combined effect of acoustic coupling constant and thermal relaxation time. The total attenuation in Ho is greater than the others; hence its ductility will be least in comparison to other lanthanides. The ultrasonic attenuation due to Akhieser damping is received large in comparison to other hexagonal structured materials due to being their large acoustic coupling constant and low higher order elastic constant $[9,10]$. These metals can be classified on the basis of the ultrasonic attenuation and thermal relaxation time because these physical quantities are quite predictable for them.

\section{CONCLUSIONS}

On the basis of above discussion, we conclude that:

- Guo and Wang [23] used gradient-correlated functional (GDF) method (ab- initio) to calculate the elastic constants of hcp structured materials. Method is accurate but very tedious, having lot of parameters and calculations. The calculation used in this method is some typical and lengthy, while in present study; we have used simple starting from Keating's approach using Lennard-Jones potential. The present method is quite simple and requires only the lattice parameter to evaluate elastic constant.

- The elastic constant and ultrasonic velocity of these metals are received similar to lave-phase compound but are found smaller than third group nitrides. Low bulk modulus indicates that a stress may cause large strain in these metals.

- The order of thermal relaxation time for these metals is found in pico second, which justifies their hep structure at $300 \mathrm{~K}$. The re-establishment time for the equilibrium distribution of thermal phonons will be minimum for the wave propagation along $\theta=55^{\circ}$ due to being smallest value of $\tau$ along this direction.

- The acoustic coupling constants of these metals for longitudinal wave are found five times larger than $\mathrm{GaN}$ /AIN/InN. Hence the conversion of acoustic energy into thermal energy will be large for these metals.

- The dominant mechanism for ultrasonic attenuation in these metals is phonon-phonon interaction. The thermal conductivity and acoustic coupling constant are the governing factors to the total attenuation.

- The mechanical and acoustic properties of these metals will not be better than the third group nitrides due to their low ultrasonic velocity, second order elastic constants and high ultrasonic attenuation.

The obtained results in this investigation can be used for further study of these metals. Our whole theoretical approach can be applied to the evaluation of ultrasonic attenuation and related parameters to study the microstructural properties of h.c.p metals

\section{APPENDIX}

When the strain $\left(e_{J}\right)$ is applied to the crystal, it causes a change in the mode frequency $\left(v_{\mathrm{I}}\right)$ given by $v_{\mathrm{I}}=v_{\mathrm{IO}}\left(1-\sum_{J=1}^{6} \gamma_{I}^{J} \mathrm{e}_{J}\right)$

where $v_{\mathrm{I} 0}$ is the frequency of the mode in the standard state.

Differentiating expression of $v_{\mathrm{I}}$, we have $\gamma_{I}^{J}=-\frac{\partial v_{I} / \partial e_{J}}{v_{I 0}}$ 
Appendix A. Equations of Grüneisen Numbers for Longitudinal Wave

\begin{tabular}{|c|c|c|}
\hline Type of Wave & No. of Modes & $\gamma_{I}^{J}$ \\
\hline Long. & 1 & $1+\left[\frac{\left(C_{13}+C_{333}\right)}{2 C_{33}}\right]$ \\
\hline Long. & 4 & {$\left[\frac{\left(C_{13}+C_{113}\right)}{2 C_{11}}\right]$} \\
\hline Shear & 4 & {$\left[\frac{\left(C_{13}+C_{344}\right)}{2 C_{44}}\right]$} \\
\hline Shear & 4 & {$\left[\frac{\left(2 C_{13}+C_{113}-C_{123}\right)}{4 C_{44}}\right]$} \\
\hline Shear & 4 & {$\left[1+\frac{\left(C_{13}+C_{344}\right)}{2 C_{44}}\right]$} \\
\hline Long. & 8 & $\frac{A}{A+C}+\frac{(A+C)}{2\left[C C_{11}+A\left(2 C_{44}+C_{13}\right)\right]} C_{13}+\frac{C^{2} C_{113}+A^{2} C_{333}+2 A C\left(C_{133}+2 C_{344}\right)}{(A+C)^{3}}$ \\
\hline Shear & 8 & $\frac{\left[(A+C) C_{13}+\frac{1}{2} C\left(C_{113}-C_{123}\right)+A C_{344}\right]}{\left[C\left(C_{11}-C_{12}\right)+2 A C_{44}\right]}$ \\
\hline Shear & 8 & $\begin{array}{l}\frac{A}{A+C}+\frac{(A+C)}{2\left[C C_{44}+A\left(C_{33}-C_{44}-C_{13}\right)\right]} \\
X\left[C_{13}+\frac{A}{(A+C)^{2}}\left(C_{113}+C_{333}-2 C_{123}\right)+\frac{(C-A)^{2}}{(C+A)^{2}} C_{344}\right]\end{array}$ \\
\hline
\end{tabular}

Appendix B. Equations of Grüneisen Numbers for Shear Wave

\begin{tabular}{|c|c|c|}
\hline Type of Wave & No. of Modes & $\gamma_{I}^{J}$ \\
\hline Long. & 1 & $L M\left(1+\frac{A\left(C_{44}+2 C_{344}\right)+C\left(C_{44}+2 C_{155}\right)}{C C_{11}+A\left(2 C_{44}+C_{13}\right)}\right)$ \\
\hline Long. & 1 & $-L M\left(1+\frac{A\left(C_{44}+2 C_{344}\right)+C\left(C_{44}+2 C_{155}\right)}{C C_{11}+A\left(2 C_{44}+C_{13}\right)}\right)$ \\
\hline Long. & 2 & $\frac{L M}{\sqrt{2}}\left(1+\frac{A\left(C_{44}+2 C_{344}\right)+C\left(C_{44}+2 C_{155}\right)}{C C_{11}+A\left(2 C_{44}+C_{13}\right)}\right)$ \\
\hline Long. & 2 & $-\frac{L M}{\sqrt{2}}\left(1+\frac{A\left(C_{44}+2 C_{344}\right)+C\left(C_{44}+2 C_{155}\right)}{C C_{11}+A\left(2 C_{44}+C_{13}\right)}\right)$ \\
\hline Shear & 2 & $-\frac{\sqrt{A C}\left(C_{155}+2 C_{44}-C_{144}\right)}{C\left(C_{11}-C_{12}\right)+2 A C_{44}}$ \\
\hline Shear & 2 & $\frac{\sqrt{A C}\left(C_{155}+2 C_{44}-C_{144}\right)}{C\left(C_{11}-C_{12}\right)+2 A C_{44}}$ \\
\hline Shear & 1 & $-\frac{\sqrt{A C}}{(A+C)}\left(1+\frac{(A-C)\left(C_{344}-C_{155}\right)-(A+C) C_{44}}{C C_{44}+A\left(C_{33}-C_{44}-C_{13}\right)}\right)$ \\
\hline Shear & 1 & $\frac{\sqrt{A C}}{(A+C)}\left(1+\frac{(A-C)\left(C_{344}-C_{155}\right)-(A+C) C_{44}}{C C_{44}+A\left(C_{33}-C_{44}-C_{13}\right)}\right)$ \\
\hline Shear & 2 & $\frac{\sqrt{A C}}{(A+C) \sqrt{2}}\left(1+\frac{(A-C)\left(C_{344}-C_{155}\right)-(A+C) C_{44}}{C C_{44}+A\left(C_{33}-C_{44}-C_{13}\right)}\right.$ \\
\hline Shear & 2 & $\frac{\sqrt{A C}}{(A+C) \sqrt{2}}\left(1+\frac{(A-C)\left(C_{344}-C_{155}\right)-(A+C) C_{44}}{C C_{44}+A\left(C_{33}-C_{44}-C_{13}\right)}\right.$ \\
\hline
\end{tabular}

here $\mathrm{A}=\left(\mathrm{C}_{11}-2 \mathrm{C}_{44}-\mathrm{C}_{13}\right)$ and $\mathrm{C}=\left(\mathrm{C}_{33}-2 \mathrm{C}_{44}-\mathrm{C}_{13}\right), \mathrm{L}=\mathrm{C} /(\mathrm{A}+\mathrm{C}), \mathrm{M}=\mathrm{A} /(\mathrm{A}+\mathrm{C})$. 
Grüneisen parameter, named after Eduard Grüneisen, describe the alteration in a crystal lattice's vibration frequency (phonon) based on the lattice increase or decrease in volume as a result of temperature change. The general expression of Grüneisen number under anisotropic Debye approximation has been derived by $\mathrm{K}$. Brugger and is written as [24]:

$-\gamma_{I}^{J}=-\gamma_{I}^{x y}=u_{x} u_{y}+\left(\frac{N_{p} N_{q}}{2 C}\right)\left(\mathrm{C}_{\text {xypq }}+u_{r} u_{s} \mathrm{C}_{\text {xypqrs }}\right)$

here superscript $x y$ is the two index symbol for the strain $e_{J}$. $N_{p}$ and $N_{q}$ are the direction cosine for the propagation direction, $u_{x}$ and $u_{y}$ are the direction cosine for the particle displacements, $C_{x y p q}$ are the second order elastic constants in tensor notation, $C_{x y p q r s}$ are the third order elastic moduli in tensor notation, $C$ is the elastic modulus controlling wave propagation in the direction determined by $N_{p}$ and $N_{q}$ and by the particle motions $u_{p}$ and $u_{\mathrm{s}}$.

Solving the Brugger's expression of $\gamma_{I}^{J}$ for the propagation of wave along unique axis $(<001>)$, the expression of Grüneisen numbers for longitudinal $\left(\gamma_{I}^{33}\right)$ and shear $\left(\gamma_{I}^{31}\right)$ wave can be obtained. The formulations are listed above (Appendix A, B).

\section{ACKNOWLEDGEMENTS}

Two of the authors (PKY and DS) are thankful to Prof. B.P.Singh, Senior Director; and Prof. Prem Prakash, Director, Amity School of Engineering \& Technology, Bijwasan, New Delhi, India for their constant encouragement to complete the work.

\section{REFERENCES}

[1] Buitrago B, Irausquin I, Mendoza J. Ultrasonic evaluation of a beta-C titanium alloy. In: Proceedings of the $4^{\text {th }}$ International Conference on Non-destructive Testing; Chania, Crete-Greece, 1114 October, 2007.

[2] Yadawa PK, Yadav RR. Ultrasonic study of intermediate-valent intermetallic $\mathrm{YbAl}_{2}$ at different temperature. Multidiscipline Model Mater Struct 2009; 5: 59-76.

[3] Kor SK, Pandey G, Singh D. Ultrasonic attenuation in lanthanum monochalcogenides from $5 \mathrm{~K}$ to $500 \mathrm{~K}$. Acta Acoustic United Acoustic 2003; 89: 105-9.
[4] Mason WP. Effect of impurities and phonon processes on the ultrasonic attenuation of germanium crystal, quartz and silicon. Physical acoustics. New York: Academic Press 1965; vol. III B: pp. 237-86.

[5] http://www.chemtopics.com/elements/lan/lan.htm. [accessed on 15 February, 2009].

[6] Landau LD, Lifshitz EM. Statistical physics. New York: Pergamon Press 1958.

[7] Yadav AK, Yadav RR, Pandey DK, Singh D. Ultrasonic study of fission products precipitated in the nuclear fuel. Mater Lett 2008; 62: 3258-61.

[8] Oligschleger C, Jones RO, Reimann SM, Schober HR. Model interatomic potential for simulations in selenium. Phys Rev B 1996; 53: 6165-73.

[9] Pandey DK, Singh D, Yadav RR. Ultrasonic wave propagation in IIIrd group nitrides. Appl Acoust 2007; 68: 766-77.

[10] Pandey DK, Yadawa PK, Yadav RR. Acoustic wave propagation in laves-phase compounds. Mater Lett 2007; 61: 4747-51.

[11] http://www.environmentalchemistry.com [accessed on 20 Jan 2009].

[12] Pandey DK, Yadawa PK, Yadav RR. Ultrasonic properties of hexagonal ZnS at nanoscale. Mater Lett 2007; 61: 5194-8.

[13] Rosen M. Elastic moduli and ultrasonic attenuation of Gd, Tb, Dy, Ho and Eb from 4.2 to 300K. Phys Rev 1968; 174: 504-14.

[14] Palmer SB, Lee EW. The Elastic constants of dysprosium and holmium. Proc R Soc Lond A 1972; 327: 519-43.

[15] Aguayo A, Murrieta G, de Coss R. Elastic stability and electronic structure of fec Ti, Zr, and Hf: a first principles study. Phys Rev B 2002; 65: 092106.

[16] Young DA. Phase diagrams of the elements. Berkely, CA: University of California Press 1991.

[17] Okamoto NL, Kusakari M, Tanaka K, Lnui H, Yamaguchi M, Otani $\mathrm{S}$. Temperature dependence of thermal expansion and elastic constants of single crystals of $\mathrm{ZrB}_{2}$ and the suitability of $\mathrm{ZrB}_{2}$ as a substrate for GaN film. J Appl Phys 2003; 93: 88.

[18] Clarke AE, Desavage BF, Bozorth R. Anomalous thermal expansion and magnetostriction of single-crystal dysprosium. Phys Rev 1965; 138: 216-24.

[19] Rhyne JJ, Legvold H, Rodine ET. Anomalous thermal expansion and magnetostriction of holmium single crystals. Phys Rev 1967; 154: $266-9$.

[20] Kor SK, Kailash. Ultrasonic attenuation study in noble metals. Indian J Pure Appl Phys 1986; 24: 179-82.

[21] Singh D, Pandey DK, Yadawa PK. Ultrasonic wave propagation in rare-earth monochalcogenides. Cent Eur J Phys 2009; 7: 198-209.

[22] Jasiukiewicz CZ, Karpus V. Debye temperature of cubic crystals Solid State Commun 2003; 128: 167-9.

[23] Guo GY, Wang HH. Gradient-corrected density functional calculation of elastic constants of Fe, Co and $\mathrm{Ni}$ in bcc, fcc and hep structures. Chin J Phys 2000; 38: 949-61.

[24] Brugger K. Physical acoustics: Principles and methods. New York: Academic Press 1965; vol. I. 